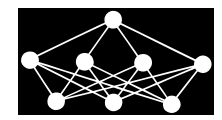

\title{
EVALUATION OF DIFFERENT PEAK MODELS OF EYE BLINK EEG FOR SIGNAL PEAK DETECTION USING ARTIFICIAL NEURAL NETWORK
}

\author{
A. Adam, Z. Ibrahim $\dagger$ N. Mokhtar,, M.I. Shapiai $\ddagger$ M. Mubin*
}

\begin{abstract}
There is a growing interest of research being conducted on detecting eye blink to assist physically impaired people for verbal communication and controlling devices using electroencephalogram (EEG) signal. One particular eye blink can be determined from use of peak points. Therefore, the purpose of peak detection algorithm is to distinguish an actual peak location from a list of peak candidates. The need of a good peak model is important in ensuring a satisfy classification performance. In general, there are various peak models available in literature, which have been tested in several peak detection algorithms. In this study, performance evaluation of the existing peak models is conducted based on Artificial Neural Network (ANN) with particle swarm optimization (PSO) as learning algorithm. This study evaluates the performance of eye blink EEG signal peak detection algorithm for four different peak models which are Dumpala's, Acir's, Liu's, and Dingle's peak models. To generalize the performance evaluation, two case studies of eye blink EEG signal are considered, which are single and double eye blink signals. It has been observed that the best test performance, in average, is $91.94 \%$ and $87.47 \%$ for single and double eye blink signals, respectively. These results indicate that the Acir's peak model offers high accuracy of peak detection for the two eye blink EEG signals as compared to other peak models. The result of statistical analysis also indicates that the Acir's peak model is better than Dingle's and Dumpala's peak models.
\end{abstract}

Key words: electroencephalogram (EEG), eye blink, peak detection algorithm, artificial neural network (ANN) classifier, particle swarm optimization (PSO), biomedical and clinical applications

Received: August 26, 2014

DOI: $10.14311 / \mathrm{NNW} .2016 .26 .004$

Revised and accepted: March 1, 2015

*Asrul Adam, Norrima Mokhtar - Corresponding author, Marizan Mubin, Applied Control and Robotics (ACR) Laboratory, Department of Electrical Engineering, Faculty of Engineering, University of Malaya, 50603 Kuala Lumpur, Malaysia, E-mail: asrul.adam@siswa.um.edu.my, norrimamokhtar@um.edu.my, marizan@um.edu.my

$\dagger$ Zuwairie Ibrahim, Faculty of Electrical and Electronic Engineering, Universiti Malaysia Pahang, 26600 Pekan, Pahang, Malaysia, E-mail: zuwairie@ump.edu.my

${ }^{\ddagger}$ Mohd Ibrahim Shapiai, Malaysia-Japan International Institute of Technology, Universiti Teknologi Malaysia Kuala Lumpur, Jalan Semarak, 54100 Kuala Lumpur, Malaysia, E-mail: md_ibrahim83@utm.my

(C)CTU FTS 2016 


\section{Introduction}

There is a growing interest of research being conducted in biological signals on detecting eye blink to assist physically impaired people for verbal communication and controlling devices. Healthy people also use this signal for vehicle accident prevention. Biological signals are defined as an electrical signal that responses to any biological activities in a human body. The biological signals that can be used to measure eye blink are electrooculogram (EOG) and electroencephalogram (EEG) signals.

To record an EOG signal, a number of electrode sensors are placed around the eyes while, to record an EEG signal, a number of electrode sensors are placed on the surface of the brain. The electrode sensors that are placed on the surface of the brain are more relevant than the electrode sensors that are placed around the eyes [12]. Based on a literature study, a number of EOG eye blink signals have widely been proposed to control cursor movement [34], a robotic arm [35], and wheelchair [9]. However, study's on the EEG eye blink signals are still limited. According to previous studies, the eye blink generated by EEG are considered as ocular artifacts [37]. Several algorithms have been developed for removing the artifacts (see [16] for review). However, the artifacts were found to be usable signals [43].

The observation of the eye blink of EEG signal indicates that the most observable signal pattern exists is peak points which signify the vertical eye movement response through the brain. A peak point is a point that holds the maximum value located on a specific time and location on an EEG signal. The known peak locations through the response of eye blink can be translated into an output, for example, wheelchair movement. Therefore, this study utilizes the peak detection algorithm to identify eye blinks on EEG signal.

A review of peak detection algorithms that is employed to the EEG signal is presented in [46]. To date, various approaches of peak detection algorithms have been proposed. These algorithms can be categorized into four main approaches based on time $[2,3,5,10,13,14,28,30,31,47]$, frequency [24], time-frequency [28, 38], and nonlinear [36] domains. In time domain approach, the peaks are analyzed as temporal information. In frequency domain approach, the peaks are analyzed as spectral information. Meanwhile, in time-frequency domain approach, the peaks are analyzed simultaneously as temporal and spectral information. In nonlinear domain approach, some statistical parameters of the peaks are analyzed.

The general peak detection algorithm usually involves several processes which are signal pre-processing, peak candidate detection, feature extraction, and classification. Various signal pre-processing methods have been employed such as data compression [11], wavelet transform [20], Kalman filter [33], and Hilbert transform [31]. Two methods for peak candidate detection have been used which are three point sliding window method [14] and k-point nonlinear energy operator (k-NEO) method [29]. Various feature extraction techniques have been proposed which are model-based [29], wavelet analysis [44], template matching [23], and power spectra analysis [15]. Several classifiers have been used, which are rule-based [14, 15], artificial neural network (ANN) $[1,3,22,28]$, support vector machine (SVM) $[2,17]$, and expert system [28]. 
Adam A. et al.: Evaluation of different peak models of eye blink EEG for...

Based on the literature, most of the researchers focus on the problem of an epileptic EEG signal when detecting peak. However, this study investigates the peak detection problem on eye blink EEG signal. Moreover, in time domain approach, 14 different peak features are derived from different peak models $[2,13$, 14, 28]. The peak model is a set of peak features that represents a peak by its amplitude, width, and slope. Most algorithms [2, 3, 13, 14, 28-30, 47] in time domain approach consider different peak models and the different styles of algorithm. Note that although different peak models have been employed for various algorithms, there are not any peak detection algorithms that find the best peak model. The use of the best peak detection model will give a chance for the algorithm to achieve the best performance. On the other hand, the chosen peak model is not necessarily suitable for different types of biological signal. Moreover, the best peak model represents some meaningful information on the signal to be evaluated.

The main objective of this study is to evaluate the performance of eye blink EEG signal peak detection algorithm on four different peak models in time domain approach. This study also highlights the significance of the best peak model. In this study, the peak detection involves the following processes: peak candidate detection, feature extraction, and classification. The sliding three-point window is a method in peak candidate detection where it is based on local maximizes to find the peak candidates. For the feature extraction method, 14 different peak features of peak candidate are calculated. The identified peak candidates are classified between true peak point and true non-peak point using ANN classifier. The particle swarm optimization (PSO) algorithm is employed as training ANN algorithm. To evaluate the performance of the algorithm, four different sets of peak feature are considered and the best peak model is suggested.

The next section briefly describes the existing peak models in time domain analysis for peak detection algorithm. Section 3 explains in details the methodology of the proposed peak detection algorithm. The experiment setup and experiment protocols are introduced in Section 4. The experimental results and discussions are presented in Section 5. Section 6 are conclusions.

\subsection{Peak model in time domain analysis}

The earliest peak model was introduced by Dumpala et al. in 1982 [14]. The peak model comprises four features, which are (1) the amplitude between the magnitude of peak point and the magnitude of valley point at the first half wave, (2) the width between valley point of first half point and valley point at second half wave, (3) and (4) two slopes between a peak point and valley point in the first half wave and second half wave. A similar definition of the peak amplitude and slopes are also been used in $[2,3,47]$.

An additional feature of peak amplitude and two features of peak width have been introduced by Acir et al. [2,3]. The additional peak amplitude is the amplitude between the magnitude of peak point and the magnitude of valley point of the second half wave. The peak widths are the width between peak point and valley point of first half wave and second half wave. The total features that are introduced by Acir et al. are six features. Acir et al. did not use the width feature that was introduced by Dumpala et al. A similar definition of the peak amplitudes, 
widths, and slopes are also been used in [29]. In [29], an additional peak feature is added with a set of feature that is introduced in $[2,3]$, which is the area of peak. However, the definition of area integration is not presented in the paper.

In addition, Liu et al. [28] have introduced 11 peak features. The peak model consists of four amplitudes: (1) the amplitude between the magnitude of peak point and the magnitude of valley point at the first half wave; (2) the amplitude between the magnitude of peak point and the magnitude of valley point of the second half wave; (3) the amplitude between the magnitude of peak and the magnitude of turning point at the first half wave, and (4) the amplitude between the magnitude of peak and the magnitude of turning point at the second half wave. The turning point is defined as the point where the slope decreases more than $50 \%$ as compared to the slope of the preceding point. The model also consists of three widths: (1) the width between valley point at first half point and valley point at second half wave, (2) the width between turning point at first half wave and turning point at second half wave, and (3) the width between half point at first half wave and half point at second half wave. Four slopes are also measured: (1) and (2) two slopes between a peak point and valley point in the first half wave and second half wave, (3) and (4) two slopes between peak point and turning point at first half wave and second half wave.

Another peak model consists of four features, which has been introduced by Dingle et al. [13]. The peak amplitude is the difference between the peak point and the floating mean. The floating mean is the average EEG which is centered at the peak point that is also called moving average curve (MAC) [30]. The width is calculated based on the difference between the valley point at the first half wave and the valley point at the second half wave. The two slopes are the slopes between a peak point and valley point in the first half wave and second half wave.

Generally, all the authors claimed that the selected peak features offer good classification performance on the proposed algorithm. However, the previous works did not provide the justification on the selected features.

\subsection{Methodology}

The EEG signals peak detection algorithm is shown in Fig. 1. In this algorithm, the peak detection involves training and testing phases. Filtered training and testing signals with known peak points is used in the training and testing phases. The first stage in peak detection is the detection of peak candidate. The next stage is extraction of the peak features based on the selected peak model. The numbers of peak features are depend on the selected peak model. Peak features of all peak candidates are extracted in this stage. Then, the peak features act as input to the ANN classifier. In the training phase, the classifier is trained to achieve the highest classification performance. During the training, the weights and threshold value in the network are varied in order to find the optimal value using the PSO algorithm approach. The optimal weights and decision threshold are then used during the testing phase. The final output of the training and testing phase is the predicted peak points and non-peak points of the identified peak candidates. 
Adam A. et al.: Evaluation of different peak models of eye blink EEG for...

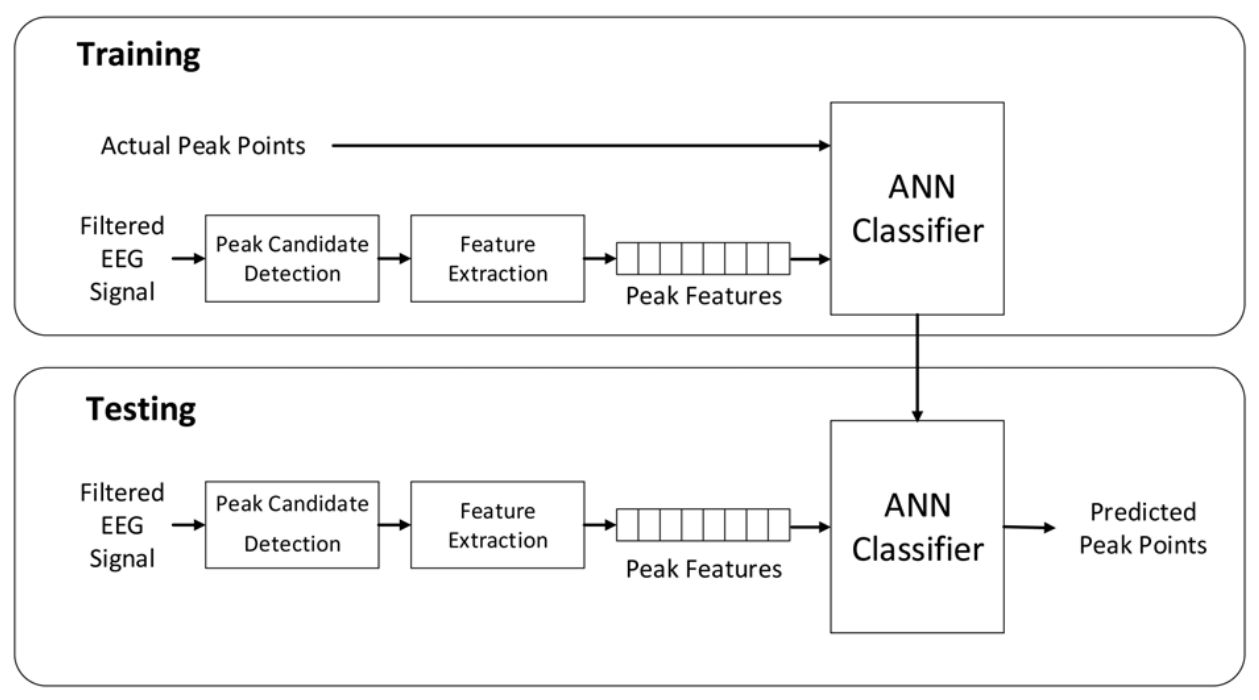

Fig. 1 The eye blink EEG signal peak detection algorithm.

\subsection{Peak candidate detection}

The first step to detect peaks is to find candidate peaks. Consider a discretetime signal, $x(I)$, of $L$ points. The $i$-th candidate peak point, $\mathrm{PP}_{i}$, are identified using three-points sliding window method [14]. Those three-points are denoted as $x(I-1), x(I)$, and $x(I+1)$ for $I=1,2, \ldots, L$. A candidate peak point is identified when $x\left(\mathrm{PP}_{i}-1\right)<x\left(\mathrm{PP}_{i}\right)>x\left(\mathrm{PP}_{i}+1\right)$ and two associated valley points, $\mathrm{VP} 1_{i}$ and $\mathrm{VP} 2_{i}$, are in between as shown in Fig. 2. Both valley points exist when $x\left(\mathrm{VP} 1_{i}-1\right)>x\left(\mathrm{VP} 1_{i}\right)<x\left(\mathrm{VP} 1_{i}+1\right)$ and $x\left(\mathrm{VP} 2_{i}-1\right)>x\left(\mathrm{VP} 2_{i}\right)<x\left(\mathrm{VP} 2_{i}+1\right)$.

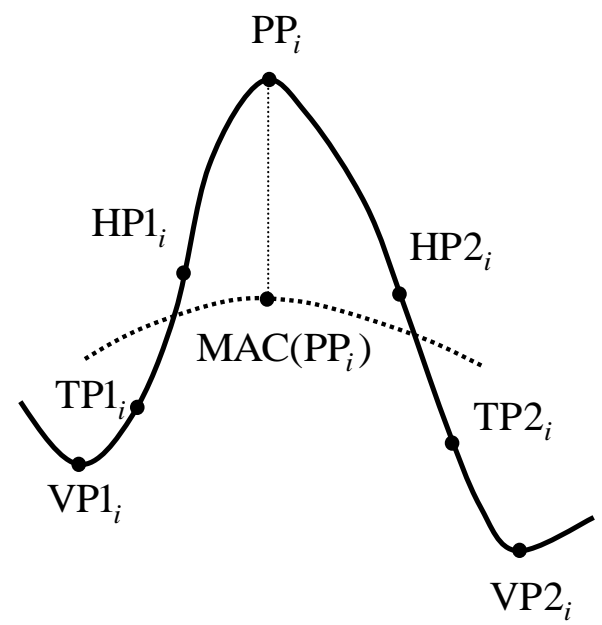

Fig. 2 Peak model parameters. 


\subsection{Feature extraction}

Based on the existing peak models, the total peak features are 14. The peak features of a peak candidate are calculated based on the eight parameters as shown in Fig. 2. The parameters consist of the $i$-th candidate peak point $\left(\mathrm{PP}_{i}\right)$, the two associated valley points $\left(\mathrm{VP} 1_{i}\right)$ and $\left(\mathrm{VP} 2_{i}\right)$, the half point at first half wave $\left(\mathrm{HP} 1_{i}\right)$, the half point at second half wave $\left(\mathrm{HP} 2_{i}\right)$, the turning point at first half wave $\left(\mathrm{TP} 1_{i}\right)$, the turning point at second half wave $\left(\mathrm{TP} 2_{i}\right)$, and the moving average curve $\left(\mathrm{MAC}\left(\mathrm{PP}_{i}\right)\right)$. The peak features can be categorized into three following groups: amplitude, width, and slope. There are five different amplitudes, five different widths, and four different slopes that can be calculated based on the parameters. All equations and description of peak features are tabulated in Tab. I. The list of different peak models and their sets of feature are tabulated in Tab. II.

\subsection{Artificial neural network classifier}

The architecture of a single layer feedforward ANN is shown in Fig. 3. In the network architecture, the input weights $w_{i j}$ are located on the connection between the input layer and the hidden layer, while the output weights, $w_{j k}$ are located on the link between the hidden layer and the output layer. The total number of weights is dependent on the number of inputs, $n$, number of outputs, $m$, and number of neurons, $l$, which can be calculated using the following equation:

$$
\text { total weights }=(n \times l)+(l \times m) \text {. }
$$

As shown in Fig. 3, hyperbolic tangent (tanh) function is chosen for all neurons at hidden and linear function for neuron in the output layer. The output of each neuron at hidden layer is denoted as $y_{j}$ and the output of neuron at output layer is denoted as $y_{k}$. The output, $y$ is a classifier output. The outputs, $y_{j}, y_{k}$, and $y$ can be defined as follows:

$$
y= \begin{cases}0 & \text { if } y_{k} \leq \theta \\ 1 & \text { if } y_{k}>\theta\end{cases}
$$

where

$$
\begin{gathered}
y_{k}=\tanh \left(\text { net }_{k}\right), \\
y_{j}=\tanh \left(\text { net }_{j}\right), \\
\tanh (\text { net })=\frac{\mathrm{e}^{\text {net }}-\mathrm{e}^{- \text {net }}}{\mathrm{e}^{\text {net }}+\mathrm{e}^{- \text {net }},} \\
\operatorname{net}_{k}=\sum_{j=1}^{l} y_{j} w_{j k} .
\end{gathered}
$$

Based on Eq. (2), the classifier can classify the output into two classes (output): class 0 and class 1 . The classifier produces 1 if $y_{k}$ is greater than the decision threshold, $\theta$. Otherwise, the classifier produces 0 . 
Adam A. et al.: Evaluation of different peak models of eye blink EEG for...

\begin{tabular}{|c|c|c|}
\hline $\begin{array}{l}\text { Peak } \\
\text { feature }\end{array}$ & Feature name & Description \\
\hline \multirow{5}{*}{ 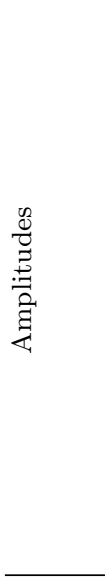 } & $\begin{array}{l}\text { Peak-to-peak ampli- } f_{1}=\left|x\left(\mathrm{PP}_{i}\right)-x\left(\mathrm{VP} 1_{i}\right)\right| \\
\text { tude at the first half }\end{array}$ & Amplitude between the magni- \\
\hline & $\begin{array}{l}\text { Peak-to-peak ampli- } f_{2}=\left|x\left(\mathrm{PP}_{i}\right)-x\left(\mathrm{VP} 2_{i}\right)\right| \\
\text { tude at the second } \\
\text { half wave }\end{array}$ & $\begin{array}{l}\text { Amplitude between the magni- } \\
\text { tude of peak and the magnitude } \\
\text { of valley of the second half wave }\end{array}$ \\
\hline & $\begin{array}{l}\text { Turning point ampli- } f_{3}=\left|x\left(\mathrm{PP}_{i}\right)-x\left(\mathrm{TP} 1_{i}\right)\right| \\
\text { tude at the first half } \\
\text { wave }\end{array}$ & $\begin{array}{l}\text { Amplitude between the magni- } \\
\text { tude of peak and the magnitude } \\
\text { of turning point at the first half } \\
\text { wave }\end{array}$ \\
\hline & $\begin{array}{l}\text { Turning point ampli- } f_{4}=\left|x\left(\mathrm{PP}_{i}\right)-x\left(\mathrm{TP} 2_{i}\right)\right| \\
\text { tude at the second } \\
\text { half wave }\end{array}$ & $\begin{array}{l}\text { Amplitude between the magni- } \\
\text { tude of peak and the magnitude } \\
\text { of turning point at the second } \\
\text { half wave }\end{array}$ \\
\hline & $\begin{array}{l}\text { Moving average am- } f_{5}=\left|x\left(\mathrm{PP}_{i}\right)-\mathrm{MAC}\left(\mathrm{PP}_{i}\right)\right| \\
\text { plitude }\end{array}$ & $\begin{array}{l}\text { Amplitude between the magni- } \\
\text { tude of peak and the magnitude } \\
\text { of moving average }\end{array}$ \\
\hline \multirow{5}{*}{ 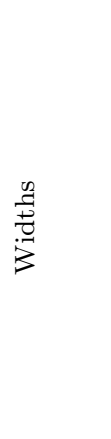 } & $f_{6}=\left|\mathrm{VP} 1_{i}-\mathrm{VP} 2_{i}\right|$ & $\begin{array}{l}\text { Width between valley point of } \\
\text { first half point and valley point } \\
\text { at second half wave }\end{array}$ \\
\hline & First half wave width $f_{7}=\left|\mathrm{PP}_{i}-\mathrm{VP} 1_{i}\right|$ & $\begin{array}{l}\text { Width between peak point and } \\
\text { valley point at first half wave }\end{array}$ \\
\hline & $\begin{array}{l}\text { Second half wave } f_{8}=\left|\mathrm{PP}_{i}-\mathrm{VP} 2_{i}\right| \\
\text { width }\end{array}$ & $\begin{array}{l}\text { Width between peak point and } \\
\text { valley point of second half wave }\end{array}$ \\
\hline & Turning point width $f_{9}=\left|\mathrm{TP} 1_{i}-\mathrm{TP} 2_{i}\right|$ & $\begin{array}{l}\text { Width between turning point at } \\
\text { first half wave and turning point } \\
\text { at the second half wave }\end{array}$ \\
\hline & $f_{10}=\left|\mathrm{HP} 1_{i}-\mathrm{HP} 2_{i}\right|$ & $\begin{array}{l}\text { Width between half point of first } \\
\text { half wave and half point of sec- } \\
\text { ond half wave }\end{array}$ \\
\hline \multirow{4}{*}{$\begin{array}{l}\bigotimes_{0}^{2} \\
\frac{0}{\sigma}\end{array}$} & $\begin{array}{l}\text { Peak slope at the first } f_{11}=\left|\frac{x\left(\mathrm{PP}_{i}\right)-x\left(\mathrm{VP} 1_{i}\right)}{\mathrm{PP}_{i}-\mathrm{VP} 1_{i}}\right| \\
\text { half wave }\end{array}$ & $\begin{array}{l}\text { Slope between a peak point and } \\
\text { valley point at the first half wave }\end{array}$ \\
\hline & $\begin{array}{l}\text { Peak slope at the sec- } f_{12}=\left|\frac{x\left(\mathrm{PP}_{i}\right)-x\left(\mathrm{VP} 2_{i}\right)}{\mathrm{PP}_{i}-\mathrm{VP} 2_{i}}\right| \\
\text { ond half wave }\end{array}$ & $\begin{array}{l}\text { Slope between a peak point and } \\
\text { valley point at the second half } \\
\text { wave }\end{array}$ \\
\hline & $\begin{array}{l}\text { Turning point slope } f_{13}=\left|\frac{x\left(\mathrm{PP}_{i}\right)-x\left(\mathrm{TP} 1_{i}\right)}{\mathrm{PP}_{i}-\mathrm{TP} 1_{i}}\right| \\
\text { at the first half wave }\end{array}$ & $\begin{array}{l}\text { The slope between peak point } \\
\text { and turning point at the first half } \\
\text { wave }\end{array}$ \\
\hline & $\begin{array}{l}\text { Turning point slope } f_{14}=\left|\frac{x\left(\mathrm{PP}_{i}\right)-x\left(\mathrm{TP} 2_{i}\right)}{\mathrm{PP}_{i}-\mathrm{TP} 2_{i}}\right| \\
\text { at the second half } \\
\text { wave }\end{array}$ & $\begin{array}{l}\text { The slope between peak point } \\
\text { and turning point at the second } \\
\text { half wave }\end{array}$ \\
\hline
\end{tabular}

Tab. I Equations and descriptions of peak features.

\subsection{Particle swarm optimization}

PSO is a stochastic population-based optimization algorithm introduced by James Kennedy and Russell Eberhart [25] in 1995. This algorithm is based on the movement and information sharing of particles in a multi-dimensional search space. The PSO algorithm has been numerously enhanced fundamentally [26, 32] and applied in many fields $[4,8,19]$. A pseudo code of the PSO algorithm is described in Algorithm 1. 


\begin{tabular}{llc}
\hline Peak model & Set of feature & No. of features \\
\hline Dumpala et al. [14] & $f_{1}, f_{6}, f_{11}, f_{12}$ & 4 \\
Acir et al. [1-3] & $f_{1}, f_{2}, f_{7}, f_{8}, f_{11}, f_{12}$ & 6 \\
Liu et al. [28] & $f_{1}, f_{2}, f_{3}, f_{4}, f_{6}, f_{9}, f_{10}, f_{11}, f_{12}, f_{13}, f_{14}$ & 11 \\
Dingle et al. [13] & $f_{5}, f_{6}, f_{11}, f_{12}$ & 4 \\
\hline
\end{tabular}

Tab. II List of different peak models and sets of feature.

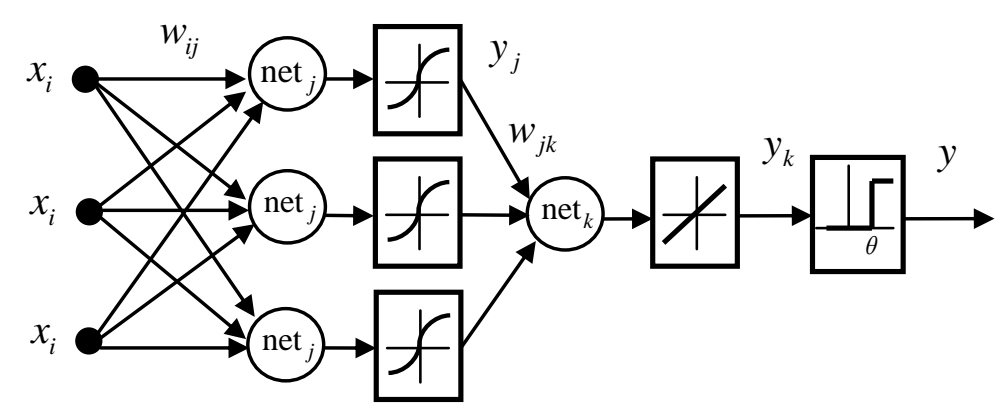

Fig. 3 Architecture of a single layer feedforward ANN.

In the preliminary stage of the PSO algorithm, some parameters are initialized: (1) PSO parameters, (2) position of particles, and (3) velocity of particles. PSO parameters include the maximum number of particles, the inertia weight, $\omega$, the cognitive component, $c_{1}$, the social component, $c_{2}$, the random values $r_{1}$ and $r_{2}$, and the maximum number of iterations, $k$. The position of particles is randomly located in the search space, and the velocity of particles is set to zero.

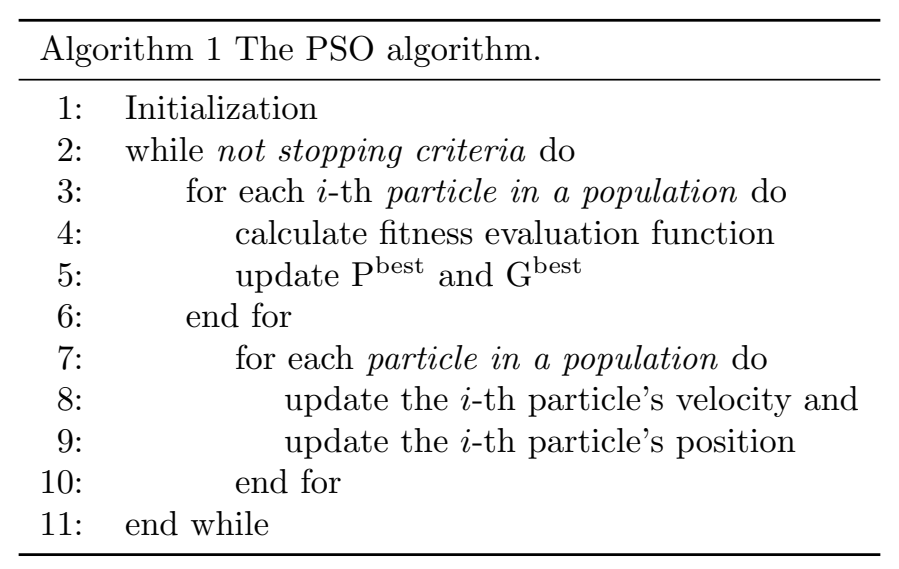

After the initialization stage is done, the fitness evaluation function is calculated. The fitness evaluation function is problem dependent. Then, the algorithm follows by updating the personal best $\left(\mathrm{P}^{\text {best }}\right)$ and the global best $\left(\mathrm{G}^{\text {best }}\right)$. The 
$\mathrm{P}^{\text {best }}$ is the best solution found by each particle, while $\mathrm{G}^{\text {best }}$ is the best solution among the $\mathrm{P}^{\text {best }}$.

After PSO algorithm progresses through the initialization and the fitness evaluation function calculation is used for updating $\mathrm{P}^{\text {best }}$ and $\mathrm{G}^{\text {best }}$, the velocities and the positions of each particle are calculated and updated. The velocity of a particle is updated using the following equation:

$$
v_{i}^{k+1}=\omega v_{i}^{k}+c_{1} r_{1}\left(\mathrm{P}^{\text {best }}-\mathrm{s}_{\mathrm{i}}^{\mathrm{k}}\right)+\mathrm{c}_{2} \mathrm{r}_{2}\left(\mathrm{G}^{\text {best }}-\mathrm{s}_{\mathrm{i}}^{\mathrm{k}}\right),
$$

where $v_{i}^{k}$ is the velocity particle $i$ at $k$-th iteration $k, r_{1}$ and $r_{2}$ are random numbers $[0,1]$, and $c_{1}$ and $c_{2}$ denote the cognitive and social coefficients, respectively. The particle's new velocity is then used to update the particle's position using following equation:

$$
s_{i}^{k+1}=s_{i}^{k}+v_{i}^{k+1},
$$

where $s_{i}^{k}$ is the position of particle $i$ at $k$-th iteration. In this study, the linear dynamic inertia weight $[40,41]$ is used and calculated as follows:

$$
\omega=\omega_{\max }-\frac{\omega_{\max }-\omega_{\min }}{k_{\max }} \times k,
$$

where $\omega_{\max }$ and $\omega_{\min }$ denote the maximum and minimum values of inertia weight, respectively, and $k_{\max }$ is the maximum iteration. The linear dynamic inertia weight takes a value between $\omega_{\max }=0.9$ and $\omega_{\min }=0.4$. Then, the PSO algorithm is terminated based on a stopping criterion. For example, if the algorithm reaches maximum iterations, then the algorithm is stopped.

The PSO algorithm adapts the neural network parameters during a training process. The primary concern is to find, during the training process, the value of the input and output weights and the decision threshold for producing the best classification performance. A process flow of ANN with the PSO algorithm for peak detection algorithm is schematically illustrated in Fig. 4.

Tab. III illustrates the representation of particle position, particle velocity, $\mathrm{P}^{\text {best }}$, and $\mathrm{G}^{\text {best }}$. The position of particle $i$ at iteration $k$ is denoted as

$$
s_{i}^{k}=\left\{w_{i, 1}^{k}, w_{i, 2}^{k}, w_{i, 3}^{k}, \ldots, w_{i, d}^{k}, w_{i, 1}^{k}, w_{i, 2}^{k}, w_{i, 3}^{k}, \ldots, w_{i, D}^{k}, \theta_{i, e}^{k}\right\},
$$

while the velocity of particle $i$ at iteration $k$ is denoted as

$$
v_{i}^{k}=\left\{v_{i, 1}^{k}, v_{i, 2}^{k}, v_{i, 3}^{k}, \ldots, v_{i, d}^{k}, v_{i, 1}^{k}, v_{i, 2}^{k}, v_{i, 3}^{k}, \ldots, v_{i, D}^{k}, v_{i, e}^{k}\right\} .
$$

The $\mathrm{P}^{\text {best }}$ of particle $i$ is represented as

$$
p b_{i}^{k}=\left\{p b_{i, 1}^{k}, p b_{i, 2}^{k}, p b_{i, 3}^{k}, \ldots, p b_{i, d}^{k}, p b_{i, 1}^{k}, p b_{i, 2}^{k}, p b_{i, 3}^{k}, \ldots, p b_{i, D}^{k}, v_{i, e}^{k}\right\}
$$

and the $\mathrm{G}^{\text {best }}$ is denoted as

$$
g b^{k}=\left\{g b_{1}^{k}, g b_{2}^{k}, g b_{3}^{k}, \ldots, g b_{d}^{k}, g b_{1}^{k}, g b_{2}^{k}, g b_{3}^{k}, \ldots, g b_{D}^{k}, g b_{e}^{k}\right\} .
$$

The $d=1,2,3, \ldots, n l$ is a $d$-th dimension of input weights, the $D=n l+1, n l+$ $2, n l+3, \ldots, n l+l m$ is a $D$-th dimension of output weights, and the $n l+l m+1$ is an $e$-th dimension of decision threshold. $n l$ is the total number of input weights. 


\begin{tabular}{|c|c|c|c|c|c|c|c|c|}
\hline \multirow{2}{*}{\multicolumn{2}{|c|}{ Particle Notations }} & \multicolumn{7}{|c|}{ Neural network parameters } \\
\hline & & \multicolumn{3}{|c|}{$\begin{array}{l}\text { Input } \\
\text { weights }\end{array}$} & \multicolumn{3}{|c|}{$\begin{array}{l}\text { Output } \\
\text { weights }\end{array}$} & \multirow{2}{*}{$\begin{array}{c}\begin{array}{c}\text { Decision } \\
\text { threshold }\end{array} \\
n l+l m+1\end{array}$} \\
\hline \multicolumn{2}{|c|}{ Dimension } & 1 & 2 & $\ldots n l$ & $n l+1$ & $n l+2$ & $\ldots n l+l m$ & \\
\hline Position & $s_{i}^{k}$ & $w_{i, 1}^{k}$ & $w_{i, 2}^{k}$ & $w_{i, d}^{k}$ & $w_{i, 1}^{k}$ & $w_{i, 2}^{k}$ & $w_{i, D}^{k}$ & $\theta_{i, e}^{k}$ \\
\hline Velocity & $v_{i}^{k}$ & $v_{i, 1}^{k}$ & $v_{i, 2}^{k}$ & $\ldots v_{i, d}^{k, a}$ & $v_{i, 1}^{k, 1}$ & $v_{i, 2}^{k, 2}$ & $v_{i, D}^{k, D}$ & $v_{i, e}^{k, c}$ \\
\hline $\mathrm{P}^{\text {best }}$ & $p b_{i}^{k}$ & $p b_{i, 1}^{k}$ & $p b_{i, 2}^{k}$ & $\ldots p b_{i, d}^{k}$ & $p b_{i, 1}^{k}$ & $p b_{i, 2}^{k}$ & $\ldots p b_{i, D}^{k}$ & $p b_{i, e}^{k}$ \\
\hline $\mathrm{G}^{\text {best }}$ & $g b^{k}$ & $g b_{1}^{k}$ & $g b_{2}^{k}$ & $\ldots g b_{d}^{k}$ & $g b_{1}^{k}$ & $g b_{2}^{k}$ & $\ldots g b_{D}^{k}$ & $g b_{e}^{k}$ \\
\hline
\end{tabular}

Tab. III Representation of Particle Position, Particle Velocity, $\mathrm{P}^{\mathrm{best}}$, and $\mathrm{G}^{\mathrm{best}}$.

$l m$ is the total number of output weights. The total number of weight is denoted as $n l+l m$. The total number of particle dimension is denoted as $n l+l m+1$.

The PSO fitness evaluation function is based on $G^{\text {mean }}[18]$. The $G^{\text {mean }}$ is calculated as follows:

$$
\begin{gathered}
\mathrm{TPR}=\frac{\mathrm{TP}}{\mathrm{TP}+\mathrm{FN}}, \\
\mathrm{TNR}=\frac{\mathrm{TN}}{\mathrm{TN}+\mathrm{FP}}, \\
\mathrm{G}^{\text {mean }}=\sqrt{\mathrm{TPR} \times \mathrm{TNR}},
\end{gathered}
$$

where true peak (TP) is correctly detected peak point of peak candidate, true nonpeak (TN) is correctly detected non-peak point of peak candidate, false peak (FP) is wrongly detected the non-peak point of peak candidate, false non-peak $(\mathrm{FN})$ is wrongly detected the peak point of peak candidate, TPR is a sensitivity or true peak rate, and TNR is a specificity or a true non-peak rate.

\section{Experimental setup}

The experiment is conducted in 10 independent runs. The first $50 \%$ of the filtered EEG signal is divided for training data, and the remaining $50 \%$ is for testing data. For the ANN classifier, as shown in Tab. IV, the numbers of neurons are selected using a trial and error method which is set to 10 . The hyperbolic tangent $[-1,1]$ is used as an activation function in the hidden layer for normalization, while a linear function is located inside the neuron in the output layer. Other settings for the ANN classifier, such as the number of neurons in the input layer and the total number of weights, are dependent on the dataset as calculated using Eq. (1). The PSO algorithm is employed as the learning algorithm which is to produce the optimal weights and decision threshold value. The PSO parameter values are based on the suggested values by Shi and Eberhart [42]. The parameters setting of PSO are tabulated in Tab. V. For each run, 10 particles are used, and the maximum iteration was set to 150 . 
Adam A. et al.: Evaluation of different peak models of eye blink EEG for...

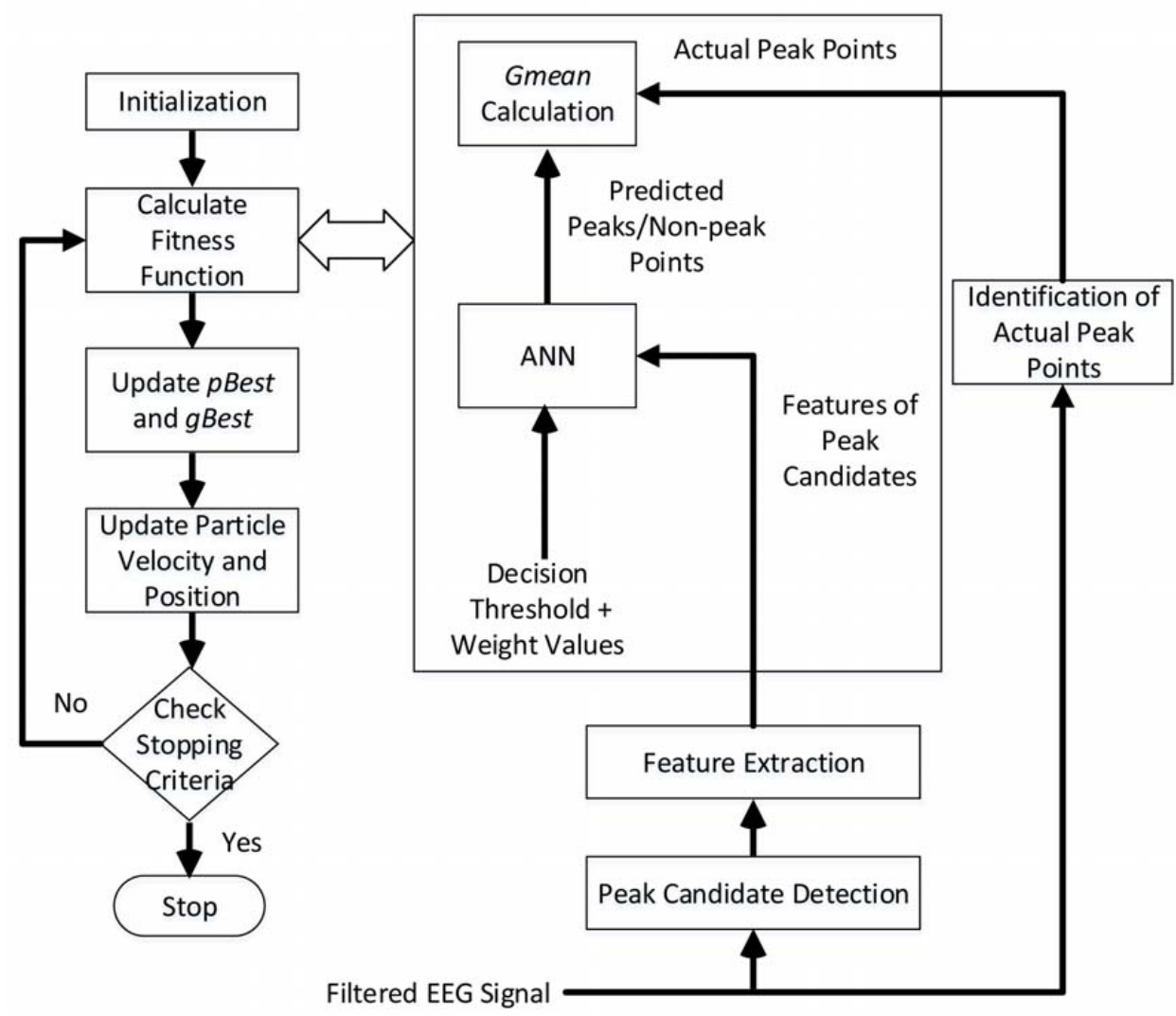

Fig. 4 ANN with the PSO algorithm for peak detection algorithm.

\begin{tabular}{ll}
\hline Parameters & Value \\
\hline Number of neuron at hidden layer & 10 \\
Activation function at hidden layer & Hyperbolic tangent $[-1,1]$ \\
Activation function at output layer & Linear function \\
Number of neurons in the input layer & $\begin{array}{l}\text { Depends on number of features } \\
\text { Total number of weights }\end{array}$ \\
\hline
\end{tabular}

Tab. IV Parameters setting of ANN.

\section{$2.1 \quad$ Experimental protocols}

This study uses two different set of eye blink EEG signals to evaluate the algorithm. The observation of the eye blink EEG signal indicates that the most observable signal pattern is the peak point which signifies the brain response on eye blink.

The experimental protocol to acquire this EEG signal was reviewed and approved by the Medical Ethic Committee (MEC) in the University of Malaya Medical Centre (UMMC). The subject gave a written consent prior to the data collection 
session. This EEG signal was acquired in the Applied Control and Robotic (ACR) Laboratory, Department of Electrical Engineering, Faculty of Engineering, University of Malaya, Malaysia. 30 healthy subjects were involved voluntarily in these data collection sessions who were undergraduate and postgraduate students in the Faculty of Engineering.

The filtered EEG signal recording was conducted using the g.USBamp biological signal acquisition system. The EEG sensors are a number of electrodes which are placed using the 10-20 international electrode placement system. The EEG signal was recorded from F9 channel. The reference electrode was located on the ear. The ground electrode was located on channel AFz. The sampling frequency was set to $256 \mathrm{~Hz}$.

Fig. 5 shows two different filtered EEG signals that are named as single eye blink signal, and double eye blink signal. The dotted-red vertical lines show the actual peak point. The actual peak points have been identified by EEG expert. The descriptions of both signals are tabulated in Tab. 6 .

The single eye blink signal has 30 signals, 10-second length per signal, 2560 sampling points per signal, and each signal containing two known peak points and some additional signal patterns. The additional signal patterns are the edge transitions which represent the eye movements. The known peak pattern in this signal represents a single eye blink. The peak pattern of a single eye blink is useful as an additional feature for controlling an electric wheelchair [27]. The total training and testing sampling points are 38400 and 38400, respectively. From the total sampling points, 3238 sampling point locations are identified as the locations of peak candidates, 60 sampling point locations are identified as the locations of true peaks, and 3178 sampling point locations are identified as the locations of false peaks.

The double eye blink signal has 5 signals, 80 -second length per signal, 20480 sampling points per signal, and each signal containing 8 known peak points and some additional signal patterns. The additional signal patterns are the edge transitions which represent the eye movements. Sometimes the signals containing a peak of the single eye blink. The peak pattern of the double eye blink is also useful as an additional feature for controlling a wheelchair [6]. The total training and testing sampling points are 51200 and 51200, respectively. From the total sampling points, 4662 sampling point locations are identified as the locations of peak candidates, 40 sampling point locations are identified as the locations of true peaks, and 4622 sampling point locations are identified as the locations of false peaks.

In general, the peak amplitude of EEG signal is different from one subject to another where it can vary between 600 and $1100 \mu \mathrm{V}$ [21]. Another research work by Sovierzoski, M.A., et al. [45], have analyzed the electrical behavior of EEG eye blink events. The research work has recorded the minimum, maximum, and the average of peak amplitude. The minimum value of amplitude was $55 \mu \mathrm{V}$. The maximum value of amplitude was $533 \mu \mathrm{V}$. The average of peak amplitude was $170 \mu \mathrm{V}$. These findings showed that the peak amplitude can vary from 55 up to $533 \mu \mathrm{V}$ and it is depend on subjects. Sometimes, the amplitude is higher than usual due to various noises.

In this study, the single and double eye blink signals that are shown in Figs. 5(a) and 5(b) were recorded from different subjects. It is also shown that both figures 
Adam A. et al.: Evaluation of different peak models of eye blink EEG for...

consist of different baseline. Based on this reason, the peak amplitude of double blink is much lower than the single eye blink. However, for one particular subject, the same value of peak amplitude between double and single eye blinks can be observed as shown in Fig. 5(b). Moreover, the peak amplitude can be measured for both figures, where the values are approximately around 300 to $500 \mu \mathrm{V}$.

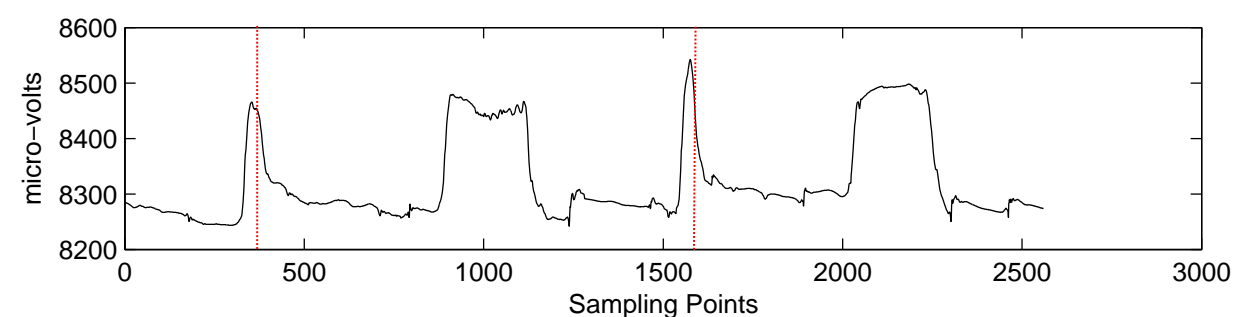

(a)

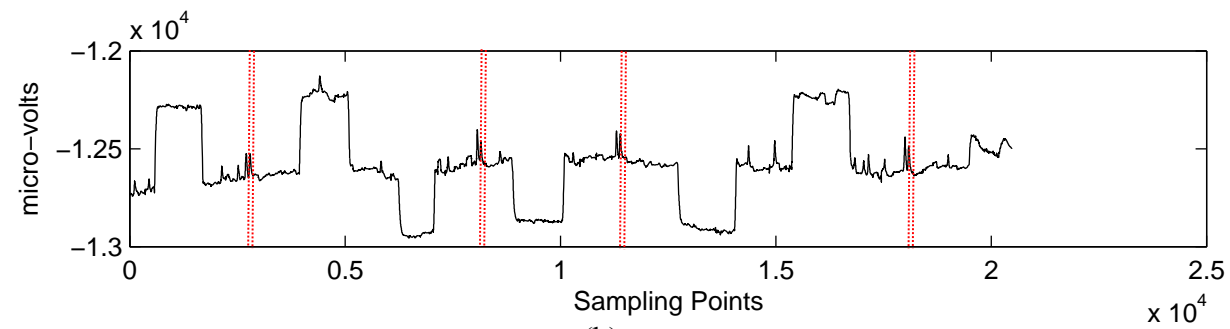

(b)

Fig. 5 The filtered EEG signal: (a) single eye blink (2 peak points per signal) and (b) double eye blink (8 peak points per signal).

\section{Experimental results and discussions}

Tab. 7 shows the performance of each peak model for a single eye blink signal. For the single eye blink signal experiments, the best average test performance is 91.94\%, which was obtained by Acir's peak model. On the other hand, the worst test performance is $73.86 \%$, which was obtained by Dingle's peak model. Based on Acir's peak model test performance, the maximum test performance reached $97.08 \%$, the minimum test performance dropped to $85.26 \%$, and the standard deviation value is $4.12 \%$. The best and worst test performance is also highlighted in the table. The sensitivity and specificity of Acir's peak model are $94.91 \%$ and $89.38 \%$, respectively. The whole sensitivity and specificity of test performance are shown in Tab. 8.

Tab. 9 shows the performance of each peak model for the double eye blink signal. Based on the end results in the Tab. 8, the best average test performance is $87.47 \%$, which was obtained by Acir's peak model. The worst test performance is $75.79 \%$, which was obtained by Dingle's peak model. Based on Acir's peak model test performance, the maximum test performance reached $92.10 \%$, the minimum test performance dropped to $82.72 \%$, and the standard deviation value is $3.17 \%$. The best and worst test performance is also highlighted in the table. The sensitivity and 


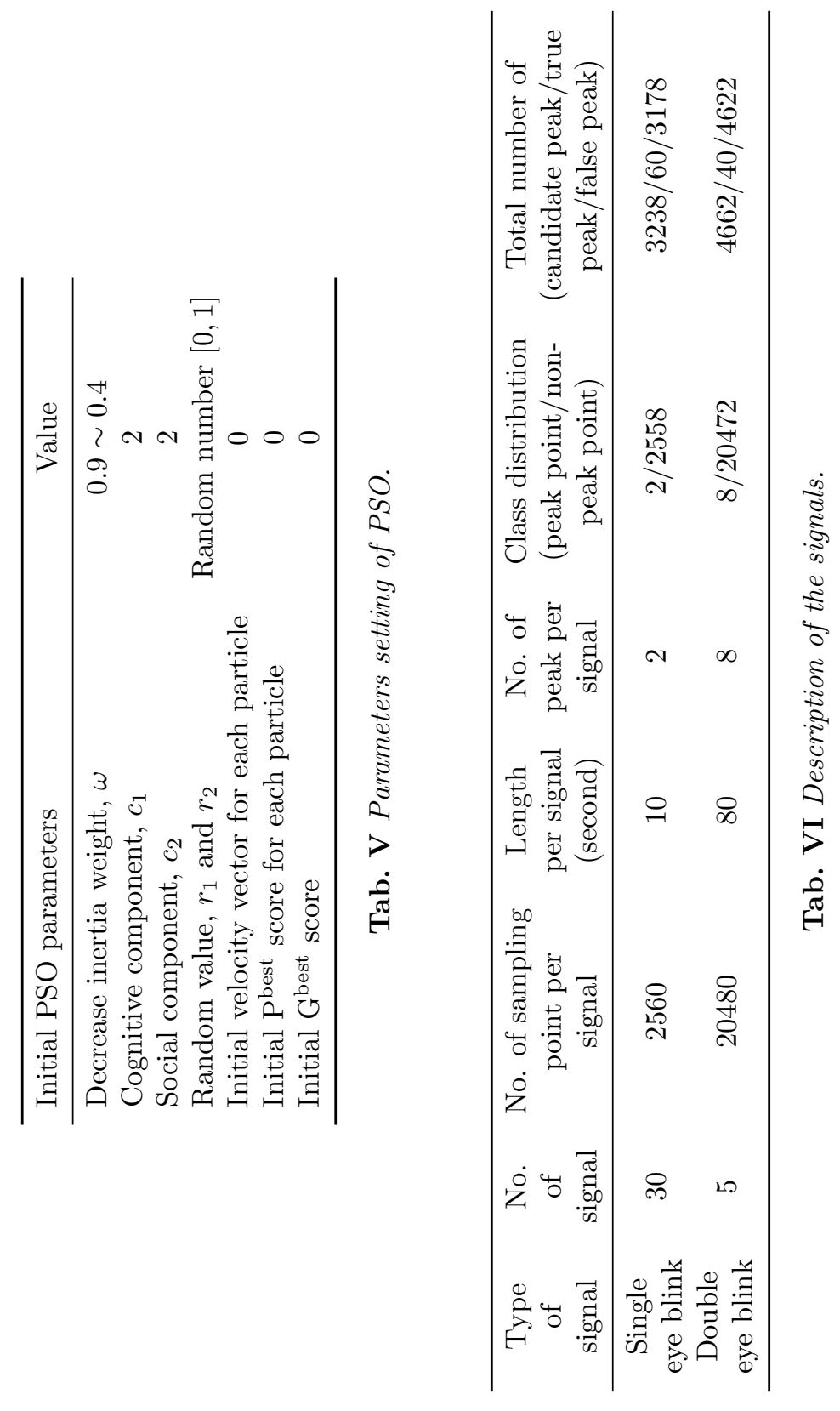


specificity of Acir's peak model are $88.74 \%$ and $86.33 \%$, respectively. The whole sensitivity and specificity of test performance for double eye blinking problem are shown in Tab. X.

Overall, Acir's peak model offers the highest classification performance rate on the single eye blink and double eye blink signals. In general, it may due to the small variation of peak width of Acir's peak model. Therefore, the small variation of peak width relaxes the learning process of the classifier in the algorithm.

Moreover, the main concern in developing any techniques resorts to the ability of generalizing the model. The main index to measure the generalization capability is best to indicate by minimum G $^{\text {mean }}$. Referring in Tab. VII and Tab. IX, Acir's peak model obtained the highest classification rate for minimum testing result on the single eye blink (85.26\%) and double eye blink (82.72\%) as compared to the Dumpala's, Liu's, and Dingle's peak models. Therefore, based on these experimental results, Acir's peak model provided a good generalized model on the single eye blink and double eye blink signals.

Also, the comparison of the average test accuracy between the four peak models is proved using Friedman's test as statistical analysis. The analysis indicates that there is a difference in test accuracies between the peak models with $p$-value is lower than 0.01 for the two signals. The average ranking of Friedman's test for the two signals is tabulated in Tab. XI. The first ranked is Acir's peak model, then it is followed by Liu's, Dumpala's, and Dingle's peak models. The posthoc analysis for Friedman's test is then evaluated to find the relationship through the comparison of the four peak models. The post-hoc analysis is based on HolmBonferroni method using two difference confidence intervals, $\alpha=0.05$ and $\alpha=0.10$. Both Friedman' test and Holm-Bonferroni post-hoc analysis are carried out by using KEEL software tool [7]. The results in Tab. XII show similar patterns for $\alpha=0.05$ and $\alpha=0.10$ for the two signals where Acir's peak model offers significantly better test accuracies than Dingle's and Dumpala's peak models. In addition, there is no significant difference in test accuracies for Acir's and Liu's peak models, Dumpala's and Dingle's peak models, and Dumpala's and Liu's peak models. In other words, the Acir's peak model is proved through statistical analysis to offer the highest accuracy as compared to previous results tabulated in Tabs. VII, VIII, IX, and X.

In this study, the ratio between true peak and false peak is 60:3178 for single eye blink signal and 40:4622 for double eye blink signal, respectively. That means both signals have extremely imbalanced dataset ratio. In this case, the conventional ANN learning algorithm such as levenberg marquart and gradient descent may fail to offer high accuracy of performance for imbalanced dataset problem. Hence, metaheuristic algorithms are more suitable to handle the training [48]. There are several metaheuristic algorithms can be employed such as PSO, genetic algorithm (GA), simulated annealing (SA), and Tabu search (TS) to train ANN for this type of problem. The PSO algorithm is used in this study because it has proved to be effective to train ANN for imbalanced dataset problem [39].

In utilizing PSO to train ANN for imbalanced dataset, the existing fitness evaluation function for balanced dataset need to be reformulated using $\mathrm{G}^{\text {mean }}$. Therefore, the key factor in solving the problem of extremely imbalanced dataset in this study relies on the capability of $G^{\text {mean }}$ as fitness evaluation function. In order to maximize the $\mathrm{G}^{\text {mean }}$, PSO provides the most appropriate ANN parameters and 


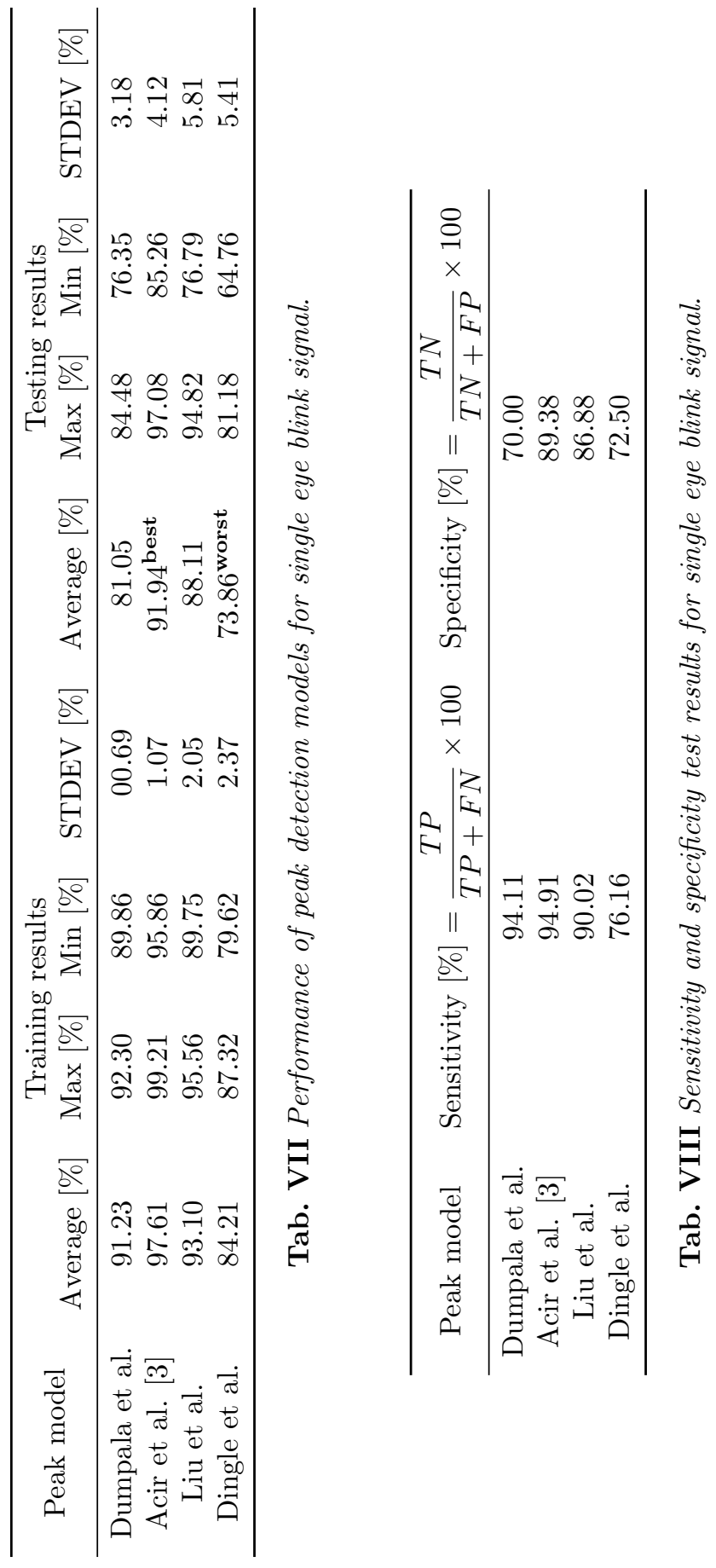


Adam A. et al.: Evaluation of different peak models of eye blink EEG for...

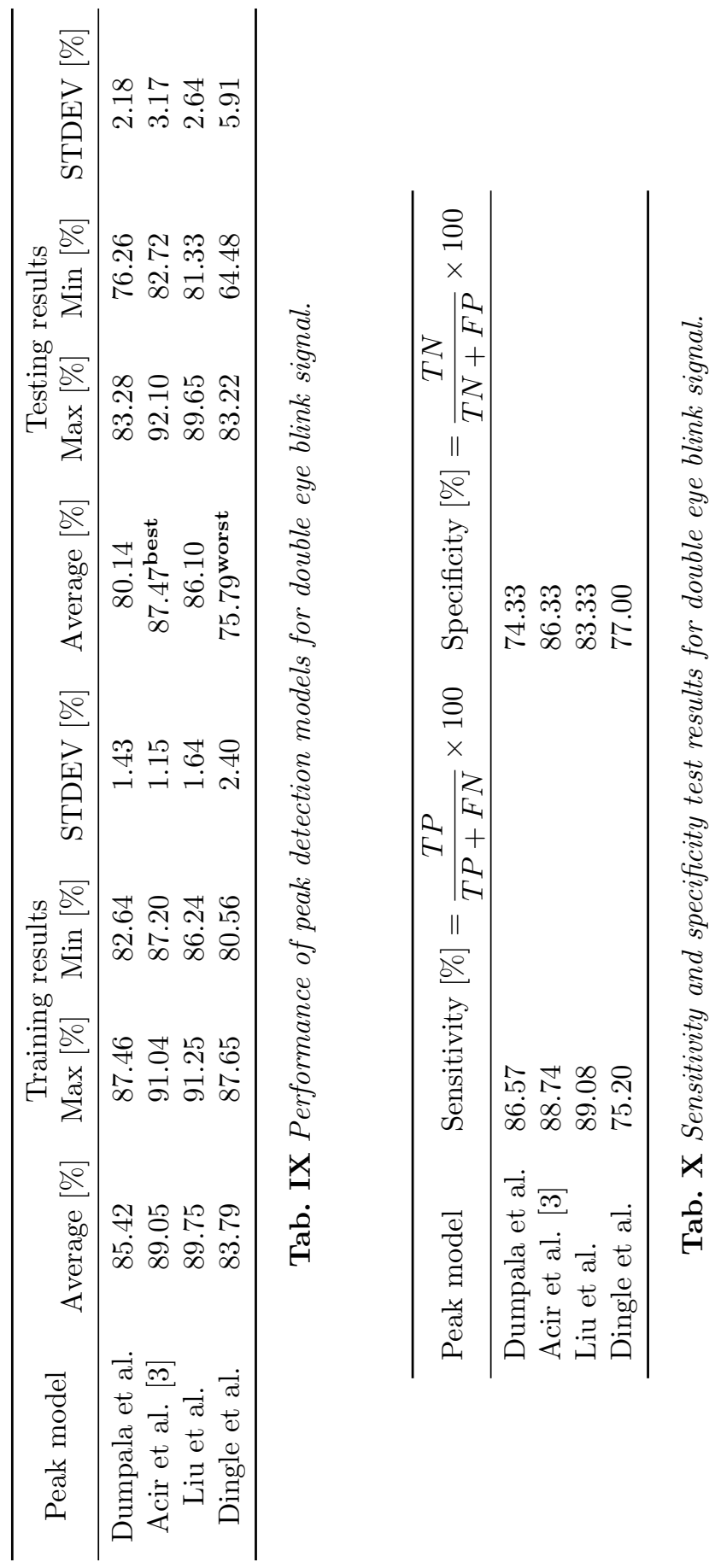



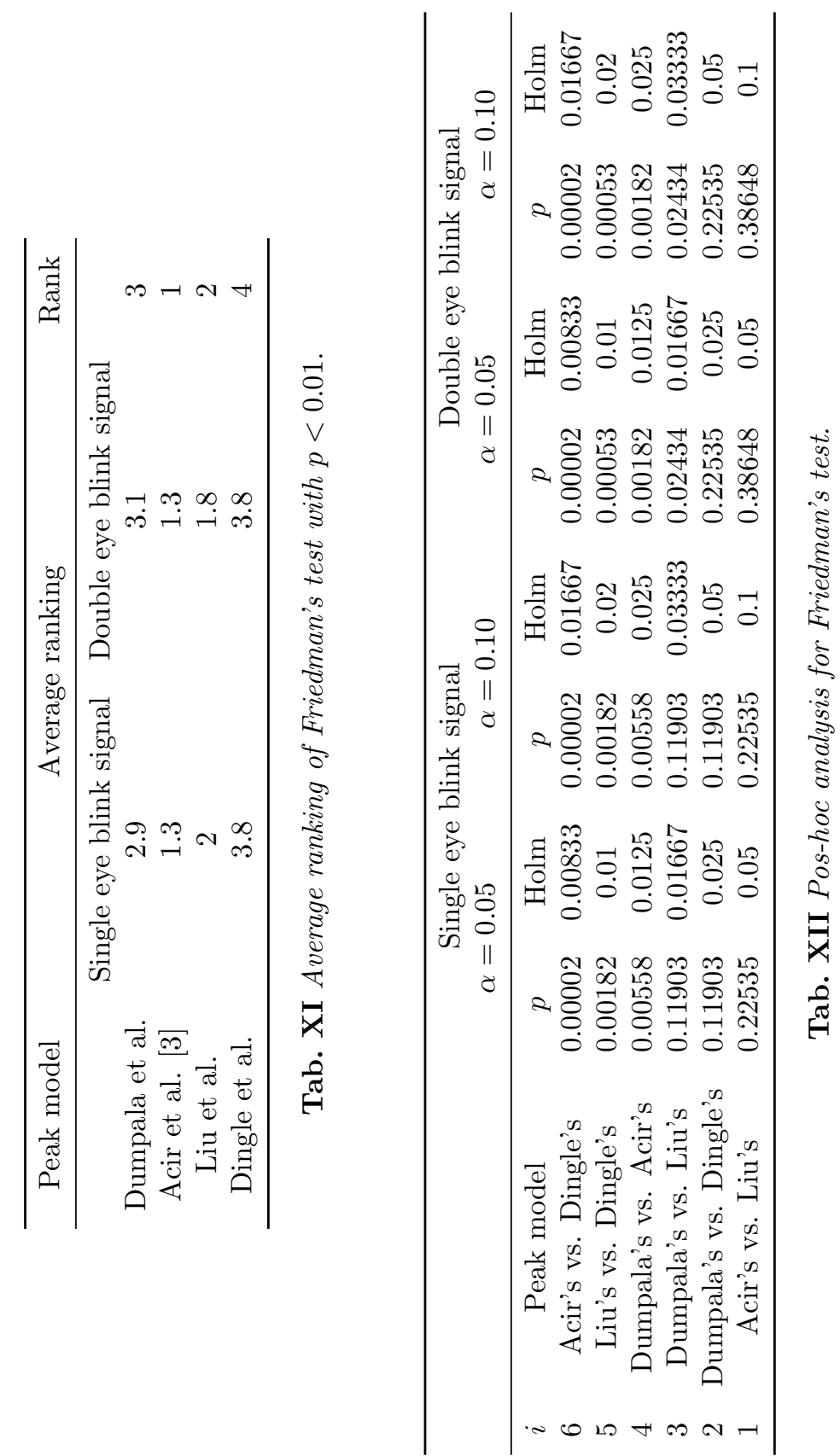
consequently balancing the detection rate between false peak and true peak rates of the dataset.

Furthermore, three peak models, which are Acir's, Liu's, and Dingle's, have been successfully employed in EEG epilepsy application while Dumpala's peak model has successfully been used for Electric Control Activity (ECA) application $[3,13,14,28]$. The utilization of a particular peak model is to detect true peaks in a particular application. For example in epilepsy detection application, when a number of true peaks are detected within certain times, an epilepsy event may occur. Similar approach is also used in eye blink detection application. When one true peak or two true peaks are detected, a subject may has blinked his eyes once or twice, respectively. This method has proved to achieve good detection performance especially for epilepsy application. As pointed out by Dumpala et al. [14], Acir et al. [3], Liu et al. [28], and Dingle et al. [13], their detection system with the selected peak model and subsequent processes contributed to the good detection performance. Although good detection performance has been achieved in $[3,13$, 14, 28], yet there used various type of peak detection algorithms on different peak models. Therefore, the need for further research on the evaluation of detection performance using combination of different types of peak models in a common platform such as ANN for fair evaluation.

Without precisely determines the peak model from the collection of features, the best detection performance only depends on the design of subsequent processes, for example, classification process. Therefore, in this study, with precisely determination of peak model, the peak detection algorithm with Acir's peak model and the subsequent processes (ANN with the PSO learning algorithm) offers good detection performance on EEG eye blink detection. Additionally, the peak model that gives the lowest detection performance can be determined as well.

This study may provide significant contribution in many applications. For example, in medical diagnostic, human machine interface (HMI), brain computer interface (BCI), and harmonic detection in digital and audio signal processing as these applications share a common peak detection problem. In devising a common platform for detecting the peak with various peak features, this study may offer more generalized solution and avoid problem dependent solution.

\section{Conclusions}

In this study, ANN with the PSO learning algorithm has been employed as a classifier for peak detection in eye blink EEG signals in order to evaluate the performance of four different peak models. The eye blink EEG signals that were collected from 30 healthy subjects were used in the investigation. From this, two case studies of eye blink signal involving single and double eye blink signals were considered for performance evaluation of all peak models. The finding of the both performance evaluations indicates that the Acir's peak model is the best peak model for eye blink EEG signals peak detection. The results indicate the highest average test performance of peak detection algorithm is $91.94 \%$ and $87.47 \%$ for single and double eye blink signals, respectively. This is due to the small variation of peak width of Acir's model that relaxes the learning process of the classifier in the algorithm. Furthermore, the results also indicate that the Acir's peak model is 
capable to provide a good generalized model by measuring the highest classification rate of minimum testing result. In statistical analysis by Friedman's test, there is a significant difference in test accuracies between the peak models for the two signals. Also, the pos-hoc analysis indicates that the Acir's peak model is better than Dingle's and Dumpala's peak models. In general, from the experimental results, it can be observed that the relevant peak model and the chosen $\mathrm{G}^{\text {mean }}$ function are the main factors in achieving higher classification rate. The utilization of the PSO learning algorithm with ANN for peak detection algorithm is to balance the ratio between false peak and true peak detection rates. As there are various combinations of peak features in the existing peak models, the use of feature selection will be investigated in future to further increase the classification rate.

\section{Acknowledgement}

This research is funded by High Impact Research Fund, UM.C/HIR/MOHE/ENG /16 Account code: D000016-16001 and Research Acculturation Grant Scheme (RDU121403) awarded by Ministry of Education Malaysia to University of Malaya and Universiti Malaysia Pahang, respectively. The first author would like to thank the Ministry of Education Malaysia for supporting his study by awarding him a MyPhD scholarship.

\section{References}

[1] ACIR N. Automated system for detection of epileptiform patterns in EEG by using a modified RBFN classifier. Expert Systems with Applications. 2005, 29(2), pp. 455-462, doi: 10.1016/ j.eswa.2005.04.040.

[2] ACIR N., GUZELI C. Automatic spike detection in EEG by a two-stage procedure based on support vector machines. Computers in Biology and Medicine. 2004, 34(7), pp. 561-575, doi: $10.1016 / \mathrm{j}$.compbiomed.2003.08.003.

[3] ACIR N., OZTURA I., KUnTAlP M., BAKlAn B., GUZELIS C. Automatic Detection of Epileptiform Events in EEG by a Three-Stage Procedure Based on Artificial Neural Networks. IEEE Transactions on Biomedical Engineering. 2005, 52(1), pp. 30-40, doi: 10.1109/TBME. 2004.839630.

[4] ADAM A., ABIDIN A.F.Z., IBRAhIM Z., HUSAIN A.R., YUSOF Z.M., IBRAHIM I. A Particle Swarm Optimization Approach to Robotic Drill Route Optimization. In: Proceedings of the Fourth Asia International Conference on Mathematical/Analytical Modelling and Computer Simulation (AMS), Kota Kinabalu, Malaysia. IEEE, 2010, pp. 60-64, doi: 10. 1109/AMS. 2010.25.

[5] ADAM A., MOKHTAR N., MUBin M., IBRAHIM Z., TUMARI M.Z.M., SHAPIAI M.I. Feature Selection and Classifier Parameter Estimation for EEG Signal Peak Detection using Gravitational Search Algorithm. In: Proceedings of the 4th International Conference on Artificial Intelligence with Applications in Engineering and Technology, Kota Kinabalu, Sabah, Malaysia. IEEE, 2014, pp. 103-108, doi: 10.1109/ICAIET.2014.26.

[6] AHMED K.S. Wheelchair Movement Control VIA Human Eye Blinks. American Journal of Biomedical Engineering. 2011, 1(1), pp. 55-58, doi: 10.5923/j . ajbe.20110101.09.

[7] ALKALÁ-Fdez J., SÁNCHEZ L., GARCÍA S., DEL JESUS M.J., VENTURA S., GARRELL J.M., OTERO J., ROMERO C., BACARDIT J., RIVAS V.M., FERNÁNDEZ J.C., HERRERA F. KEEL: a software tool to assess evolutionary algorithms for data mining problems. Soft Computing. 2009, 13(3), pp. 307-318, doi: 10.1007/s00500-008-0323-y. 
Adam A. et al.: Evaluation of different peak models of eye blink EEG for...

[8] AYOB M.N., YUSOF Z.M., ADAM A., ABIDIN A.F., IBRAHIM I., IBRAHIM Z., SUDIN S., SHAIKH-HUSIN N., HANI M.K. A Particle Swarm Optimization Approach for Routing in VLSI. In: Proceedings of the 2nd International Conference on Computational Intelligence, Communication Systems and Networks (CICSyN), Liverpool, UK. IEEE, 2010, pp. 49-53, doi: 10.1109/CICSyN.2010.42.

[9] BAREA R., BOQUETE L., BERGASA L.M., LÓPEZ E., MAZO M. Electro-oculographic guidance of a wheelchair using eye movements codification. International Journal of Robotics Research. 2003, 22(7-8), pp. 641-652, doi: 10.1177/02783649030227012.

[10] BAREA R., BOQUETE L., ORTEGA S., LÓPEZ E., RODRÍGUEZ-ASCARIZ J.M. EOGbased eye movements codification for human computer interaction. Expert Systems with Applications. 2012, 39(3), pp. 2677-2683, doi: 10.1016/j.eswa.2011.08.123.

[11] BONNER R.E., CREVASSE L., FERRER M.I., GREENFIELD J.C. A new computer program for analysis of scalar electrocardiograms. Computers and Biomedical Research. 1972, 5(6), pp. 629-653, doi: 10.1016/0010-4809(72) 90043-2.

[12] Dimigen O., SOMMER W., HOHLFELD A., JACOBS A.M., KLIEGL R. Coregistration of Eye Movements and EEG in Natural Reading: Analyses and Review. Journal of Experimental Psychology-General. 2011, 140(4), pp. 552-572, doi: 10.1037/A0023885.

[13] DINGLE A.A., JONES R.D., CARROLL G.J., FRIGHT W. A Multistage System to Detect Epileptiform Activity in the EEG. IEEE Transactions on Biomedical Engineering. 1993, 40(12), pp. 1260-1268, doi: 10.1109/10.250582.

[14] DUMPALA S.R., REDDY S.N., SARNA S.K. An algorithm for the detection of peaks in biological signals. Computer Programs in Biomedicine. 1982, 14(3), pp. 249-256, doi: 10. 1016/0010-468X (82) 90030-7.

[15] EXARCHOS T.P., TZALLAS A.T., FOTIADIS D.I., KONITSIOTIS S., GIANNOPOULOS S. EEG Transient Event Detection and Classi?cation Using Association Rules. IEEE Transaction on Information Technology in Biomedicine. 2006, 10(3), pp. 451-457, doi: 10.1109/ TITB. 2006.872067.

[16] FATOURECHI M., FATOURECHI M., BASHASHATI A., WARD R.K., BIRCH G.E. EMG and EOG artifacts in brain computer interface systems: A survey. Clinical Neurophysiology. 2007, 118(3), pp. 480-494, doi: 10.1016/j.clinph.2006.10.019.

[17] GAO J. F., YANG Y., LIN P., WANG P., ZHENG C.X. Automatic removal of eye-movement and blink artifacts from EEG signals. Brain Topogr. 2010, 23(1), pp. 105-114, doi: 10.1007/ s10548-009-0131-4.

[18] GUO X., YIN Y., DONG C., YANG G., ZHOU G. On the Class Imbalance Problem. In: Proceedings of the Proceedings of the Fourth International Conference on Natural Computation (ICNC 08), Jinan, China. IEEE, 2008, pp. 192-201, doi: 10.1109/ICNC.2008.871.

[19] IBRAHIM Z., MOKHTAR N. A DNA Sequence Design for DNA Computation Based on Binary Vector Evaluated Particle Swarm Optimization. International Journal of Unconventional Computing. 2012, 8(2), pp. 119-137.

[20] INDIRADEVI K.P., ELIAS E., SANTHIDEVI P.S., NAYAK S.D., RADHAKRISHNAN K. A multi-level wavelet approach for automatic detection of epileptic spikes in the electroencephalogram. Computers in Biology and Medicine. 2008, 38(7), pp. 805-816, doi: 10.1016/ j.compbiomed.2008.04.010.

[21] IWASAKI M., IWASAKI M., KELLINGHAUS C., ALEXOPOULOS A.V., BURGESS R.C. KUMAR A.N. HAN Y.H., LÜDERS H.O., LEIGH R.J. Effects of eyelid closure, blinks, and eye movements on the electroencephalogram. Clinical Neurophysiology. 2005, 116(4), pp. 878-885, doi: 10.1016/j.clinph.2004.11.001.

[22] JAMES C.J., JONES R.D., BONES P.J., CARROLL G.J. Detection of Epileptiform Discharges in the EEG by a Hybrid System Comprising Mimetic, Self Organized Artificial Neural Network, and Fuzzy Logic Stages. Clinical Neurophysiology. 1999, 110(12), pp. 2049-2063, doi: 10.1016/S1388-2457(99)00168-6.

[23] JI Z., WANG X., SUGI T., GOTO S., NAKAMURA M. Automatic spike detection based on real-time multi-channel template. In: Proceedings of the 4 th International Conference on Biomedical Engineering and Informatics (BMEI), Shanghai, China. IEEE, 2011, pp. 648-652, doi: 10.1109/BMEI.2011.6098388. 
[24] JUOZAPAVI A., BACEviCiUs G., BUGELSKIS D., SAMAITIENE R. EEG analysis automatic spike detection. Journal of Nonlinear Analysis: Modelling and Control. 2011, 16(4), pp. 375-386.

[25] KENNEDY J., EBERHART R. Particle Swarm Optimization. In: Proceedings of the IEEE international Conference on Neural Networks (ICW), Perth, Western Australia. IEEE, 1995, pp. 1942-1948, doi: 10.1109/ICNN.1995.488968.

[26] LIM K.S., IBRAHIM Z., BUYAMIN S., AHMAD A., NAIM F., GHAZALI K.H., MOKHTAR N. Improving Vector Evaluated Particle Swarm Optimisation by Incorporating Nondominated Solutions. The Scientific World Journal. 2013, pp. 19, doi: 10.1155/ 2013/510763.

[27] LIN J.S., YANG W. C. Wireless Brain-Computer Interface for Electric Wheelchairs with Eeg and Eye-Blinking Signals. International Journal of Innovative Computing Information and Control. 2012, 8(9), pp. 6011-6024.

[28] LIU H.S., ZHANG T., YANG F.S. A Multistage, Multimethod Approach for Automatic Detection and Classification of Epileptiform EEG. IEEE Transaction on Biomedical Engineering. 2002, 49(12), pp. 1557-1566, doi: 10.1109/TBME.2002.805477.

[29] LIU Y.-C., LIN C.C., TSAI J.J., SUN Y.N. Model-Based Spike Detection of Epileptic EEG Data. Sensors. 2013, 13, pp. 12536-12547, doi: 10.3390/s130912536.

[30] LU W.,NYSTROM M.M., PARIKH P.J., FOOSHEE D.R., HUBENSCHMIDT J.P. BRADLEY J.D., LOW D.A. A semi-automatic method for peak and valley detection in freebreathing respiratory waveforms. Med. Phys. 2006, 33(10), pp. 3634-3636, doi: 10.1118/1. 2348764.

[31] MANIKANDANA M.S., SOMAN K.P. A novel method for detecting R-peaks in electrocardiogram (ECG) signal. Biomedical Signal Processing and Control. 2012, 7(2), pp. 118-128, doi: $10.1016 / \mathrm{j}$.bspc. 2011.03 .004 .

[32] MOHAMAD M.S., OMATU S., DERIS S., YOShIOKA M., ABDUllah A., IBRAHIM Z. An enhancement of binary particle swarm optimization for gene selection in classifying cancer classes. Algorithms for Molecular Biology. 2013, 8(1), doi: 10.1186/1748-7188-8-15.

[33] OIKONOMOUA V.P., TZALLAS A.T., FOTIADIS D.I. A Kalman filter based methodology for EEG spike enhancement. Computer methods and programs in biomedicine. 2007, 85(2), pp. 101-108, doi: 10.1016/j.cmpb.2006.10.003.

[34] PAUL G.M., PAUL G.M., CAO F., TORAH R., YANG K., BEEBY S., TUDOR J. A Smart Textile Based Facial EMG and EOG Computer Interface. IEEE Sensors Journal. 2014, 14(2), pp. 393-400, doi: 10.1109/Jsen.2013.2283424.

[35] POSTELniCU C.C., TAlABA D., TOMA M.I. Controlling a Robotic Arm by Brainwaves and Eye Movement. Technological Innovation for Sustainability. 2011, 349, pp. 157-164, doi: 10.1007/978-3-642-19170-1_17.

[36] PUtignano M., INTERMiTE A., WELSCH C.P. A non-linear algorithm for current signal ?ltering and peak detection in SiPM. Journal of Instrumenatation. 2012, 7(8), pp. 1-19, doi: 10.1088/1748-0221/7/08/P08014.

[37] SAMENI R., GOUY-PAILLER C. An iterative subspace denoising algorithm for removing electroencephalogram ocular artifacts. Journal of Neuroscience Methods. 2014, 225, pp. 97105, doi: 10.1016/j.jneumeth.2014.01.024.

[38] SENHADJI L., WENDLING F. Epileptic transient detection: wavelets and time-frequency approaches. Journal of Clinical Neurophysiology. 2002, 32, pp. 175-192, doi: 10.1016/ S0987-7053(02) 00304-0.

[39] SHAMSUDIN H.C., ADAM A., SHAPIAI M.I., BASRI M.A., IBRAHIM Z., KHALID M. An Improved Two-Step Supervised Learning Artificial Neural Network for Imbalanced Dataset Problems. In: Proceedings of the 3rd International Conference on Computational Intelligence, Modelling and Simulation (CIMSiM), Langkawi, Malaysia. IEEE, 2011, pp. 108-113, doi: 10.1109/CIMSim.2011.28.

[40] SHI Y., EBERHART R.C. A Modified Particle Swarm Optimizer. In: Proceedings of the IEEE International Comference on Evolutionary Computation, Anchorage, Alaska, USA. IEEE, 1998, pp. 69-73, doi: 10.1109/ICEC.1998.699146. 
Adam A. et al.: Evaluation of different peak models of eye blink EEG for...

[41] SHI Y., EBERHART R.C. Parameter Selection in Particle Swarm Optimization. In: Proceedings of the 7th Annual Conference on Evolutionary Programming, San Diego, CA, USA. Springer Verlag, LNCS, 1998, pp. 591-601.

[42] SHI Y., EBERHART R.C. Empirical Study of Particle Swarm Optimization. In: Proceedings of the IEEE Congress on Evolutionary Computation, Washington D.C., USA. IEEE, 1999, pp. 1945-1950, doi: 10.1109/CEC.1999.785511.

[43] SINGLA R., CHAMBAYIL B., KHOSLA A., SANTOSH J. Comparison of SVM and ANN for classification of eye events in EEG. Journal of Biomedical Science and Engineering. 2011, 4(1), pp. 62-69, doi: 10.4236/jbise.2011.41008.

[44] SINNO N., TOUT K. Analysis of Epileptic Events Using Wavelet Packets. The International Arab Journal of Information Technology. 2008, 5(4), pp. 165-169.

[45] SOVIERZOSKI M.A., ARGOUD F.I., DE AZEVEDO F.M. Identifying eye blinks in EEG signal analysis. In: Proceedings of the International Conference on Information Technology and Applications in Biomedicine (ITAB), Shenzhen, China. IEEE, 2008, pp. 406-409, doi: 10.1109/ITAB. 2008.4570605.

[46] WILSON S.B., EMERSON R. Spike detection: a review and comparison of algorithms. Clinical Neurophysiology. 2002, 113(12), pp. 1873-1881, doi: 10.1016/S1388-2457(02) 00297-3.

[47] XU L., MENG M.Q., LIU R., WANG K. Robust Peak Detection of Pulse Waveform Using Height Ratio. In: Proceedings of the 30th Annual International IEEE EMBS Conference, Vancouver, British Columbia, Canada.IEEE, 2008, pp. 2856-3859, doi: 10.1109/IEMBS. 2008. 4650051.

[48] YAGHINI M., KHOSHRAFTAR M.M., FALLAHI M. A hybrid algorithm for artificial neural network training. Engineering Applications of Artificial Intelligence. 2013, 26(1), pp. 293301, doi: 10.1016/j.engappai.2012.01.023. 